\title{
EXTRAÇÃO DE FITOQUÍMICOS DO BAGAÇO DA AMORA- PRETA EMPREGANDO A TÉCNICA LIMPA DE EXTRAÇÃO COM LÍQUIDO PRESSURIZADO
}

\author{
A. P. F. MACHADO ${ }^{1}$, J. L. PASQUEL- REÁTEGUI ${ }^{1}$, G. F. BARBERO ${ }^{2}$ e J. MARTÍNEZ ${ }^{1}$ \\ ${ }^{1}$ Universidade Estadual de Campinas, Departamento de Engenharia de Alimentos \\ ${ }^{2}$ Universidade de Cádiz, Departamento de Química Analítica \\ E-mail para contato: julian@fea.unicamp.br
}

\begin{abstract}
RESUMO - A extração com líquidos pressurizados (PLE) foi usada para obter compostos fenólicos do bagaço da amora-preta nas seguintes condições: pressão (7,5 $\mathrm{MPa})$, razão $\mathrm{S} / \mathrm{F}$ (18,0 $\mathrm{Kg}$ solvente/ $\mathrm{Kg}$ resíduo fresco), tempo (30 min), temperatura $\left(60,80\right.$ e $\left.100{ }^{\circ} \mathrm{C}\right)$ e solventes (água, água acidificada a pH 2,5, etanol e mistura de etanol+água $50 \% \mathrm{v} / \mathrm{v})$. Foram avaliados o rendimento global $\left(X_{0}\right)$, fenólicos totais (FT), antocianinas monoméricas (AM) e atividade antioxidante (AA). Para FT, AM e AA houve interação entre solvente e temperatura. $\mathrm{O}$ aumento da temperatura resultou em melhores respostas, com exceção da $\mathrm{AM}$, onde houve uma leve diminuição. $\mathrm{O}$ extrato da mistura etanol+água em $100{ }^{\circ} \mathrm{C}$ obteve bons resultados $(\mathrm{FT}=7,36 \mathrm{mg}$ EAG/g resíduo fresco $(\mathrm{RF}), \mathrm{AM}=1,02 \mathrm{mg}$ EC3G/g RF, AA $=76,03 \mu \mathrm{mol} \mathrm{ET} / \mathrm{g} \mathrm{RF}$ e $X_{0}=6,33 \%$ ), sendo considerada a melhor condição PLE. Estes valores foram maiores que os obtidos pela técnica convencional (Soxhlet). Quatro antocianinas foram identificadas por UPLC-QToF-MS.
\end{abstract}

\section{INTRODUÇÃO}

O processamento de alimentos de origem vegetal, além de produzir o produto principal, que é comercializado, gera grandes quantidades de subprodutos que não são aproveitados pela indústria. Pesquisas ao longo dos últimos 20 anos revelaram que muitos destes subprodutos podem servir como uma fonte potencialmente valiosa de compostos bioativos (Wijngaard et al., 2012). Além disso, tem sido observado nos últimos anos um crescente interesse por produtos naturais, como resultado da conscientização dos consumidores na busca por uma melhor qualidade de vida e a preocupação destes com a segurança dos produtos utilizados ou ingeridos, incluindo a ingestão de produtos contendo naturais que ofereçam benefícios para o corpo. Tal preocupação está impulsionando as indústrias alimentícias, de cosméticos, farmacêuticas e químicas na obtenção e utilização de extratos vegetais que apresentem características funcionais (biológicas), corantes ou aromatizantes, com alto grau de pureza, uma vez que substâncias sintéticas, muitas vezes, são suspeitas de causar efeitos maléficos à saúde. O isolamento desses compostos naturais possibilita o aproveitamento dos subprodutos gerados na agroindústria e podem resultar em novas alternativas empresariais, além de minimizar o impacto ambiental causado pelo acúmulo desses resíduos (Balasundram et al., 2006).

A amora-preta (Rubus spp.) tem chamado a atenção de produtores e consumidores nos últimos anos, por ser uma boa fonte de compostos bioativos que oferece benefícios para a saúde. 
Os resíduos agroindustriais do processamento de amora geralmente são uma boa fonte de flavonoides, em destaque as antocianinas, que são compostos fenólicos amplamente conhecidos pela sua atividade antioxidante (Kaume et al., 2011).

A recuperação de compostos fitoquímicos a partir dos resíduos sólidos tem sido relatada utilizando tecnologias convencionais e alternativas. Os métodos convencionais geralmente consomem muito tempo, podendo degradar compostos de interesse durante o processo extrativo, e requerem grande quantidade de solventes orgânicos (como metanol, acetona e clorofórmio), que em alguns casos são prejudiciais à saúde e causam danos ambientais, havendo a necessidade de separá-los do extrato posteriormente e descartá-los de forma adequada. Assim, a técnica de extração com líquido pressurizado (PLE) ou líquido subcrítico surgiu como alternativa para a extração e fracionamento de produtos naturais, através de uma tecnologia limpa e com a possibilidade de ajustar parâmetros visando à seletividade do processo para um grupo de compostos a serem extraídos. Esta técnica permite uma extração mais rápida dos analitos em um ambiente fechado em altas pressões e temperaturas. Estas condições melhoram a solubilidade dos analitos no solvente e a cinética de dessorção a partir de matrizes, melhorando dessa forma a eficiência/rendimento de extração (Mustafa e Turner, 2011; Wijngaard et al., 2012).

Dessa forma, o presente trabalho teve como objetivo principal obter extratos ricos em compostos fenólicos a partir do bagaço da amora-preta empregando a extração com líquidos pressurizados (PLE).

\section{MÉTODOS}

\subsection{Extração PLE}

As extrações pela técnica PLE foram realizadas em uma unidade composta pelas seguintes partes: uma célula de extração aço inoxidável de $100 \mathrm{ml}$ com filtro de metal na saída; uma camisa de aquecimento elétrica usada para manter a temperatura de extração no valor definido; uma bomba de HPLC; válvulas de bloqueio e micrométrica; termopar e um medidor de pressão.

Os experimentos de PLE foram realizados em três temperaturas $\left(60,80\right.$ e $\left.100{ }^{\circ} \mathrm{C}\right)$ e com quatro diferentes solventes (água, etanol, água+etanol 50\% v/v e água acidificada a pH 2,5). Todas as outras condições do processo foram mantidas constantes (pressão $=7,5 \mathrm{MPa}$, tempo de extração de 30 minutos, a razão mássica de solvente por resíduo $=18)$, totalizando 12 condições diferentes que foram realizadas em duplicata.

\subsection{Extrações Convencionais}

As extrações convencionais pelos métodos Soxhlet e maceração foram realizadas a partir do resíduo da amora-preta com intuito de comparar os resultados obtidos por estas técnicas com aqueles obtidos na melhor condição PLE.

Extração Soxhlet: Foi feita utilizando o metanol e o etanol como solventes de extração a temperatura em torno de $40{ }^{\circ} \mathrm{C}$ com tempo de extração de 5 horas.

Maceração: Foi realizada a temperatura ambiente empregando o metanol acidificado $(0,01$ 
$\% \mathrm{v} / \mathrm{v} \mathrm{HCl}$ ) como solvente extrator com tempo de extração de 24 horas.

\subsection{Análises dos Extratos}

Os extratos obtidos por ambas as técnicas foram avaliados em termos de rendimento global de extração $\left(X_{0}\right)$ e da sua composição química (teor de fenólicos totais (FT), antocianinas monoméricas (AM), e atividade antioxidante (AA) medida pelos métodos de DPPH e ABTS). Além disso, apenas para os extratos subcríticos foram feitas a identificação e quantificação das antocianinas majoritárias e minoritárias presentes nos mesmos empregando a Cromatografia Líquida de Ultra Eficiência (UPLC - Ultra Performance Liquid Chromatography).

Rendimento global de extração: $X_{0}$ foi obtido pela razão entre massa total extraída $\left(M_{\text {extrato }}\right)$ e a massa de alimentação da matéria-prima $(F)$, de acordo com a Equação (1):

$$
X_{0}=\frac{M_{\text {extrato }}}{F} \times 100
$$

Teor de fenólicos totais: O FT dos extratos foi determinado segundo o método espectrofotométrico de Folin-Ciocalteau, descrito por Singleton e Rossi (1965), com algumas adaptações para extratos vegetais sugerido por Singleton et al. (1999). Todos os ensaios foram realizados em duplicata e os resultados foram expressos em $\mathrm{mg}$ equivalente ácido gálico (EAG)/g de resíduo fresco $(\mathrm{RF})$.

Teor de antocianinas monoméricas: AM foram quantificadas através do método do $\mathrm{pH}$ diferencial descrito por Giust e Wrolstad (2001). Os resultados foram expressos em $\mathrm{mg}$ equivalente de cianidina 3-O-glicosídeo (EC3G)/g RF. Todos os experimentos foram realizados em duplicata.

Atividade antioxidante: as AAs dos extratos foram determinadas pelos métodos de DPPH e ABTS. Todos os ensaios foram realizados em duplicatas, e os resultados foram expressos em $\mu$ mol equivalente de Trolox (ET)/g RF. No método DPPH (1,1-difenil-2-picrilhidrazil) a capacidade dos compostos antioxidantes em sequestrar o radical DPPH• foi determinada de acordo com o descrito por Brand-Williams et al. (1995). Para o método de ABTS (ácido 2,2'-azino-bis (ácido 3-etilbenzotiazolina-6-sulfônico)), a capacidade antioxidante dos extratos contra os radicais ABTS+• foi determinada como relatado por Re et al. (1999).

\subsection{Cinética de Extração PLE}

Para os estudos da cinética de extração foram construídas curvas globais de extração expressando o rendimento global de extração acumulado $\mathrm{X}_{0}(\%)$ em função do tempo de extração (min). O estudo foi feito na melhor condição PLE, nas vazões de 1,67; 3,35 e 6,70 mL/min. 


\section{RESULTADOS}

\subsection{Extração com Líquidos Pressurizados}

A Tabela 1 mostra os resultados obtidos para as diferentes condições utilizando PLE. Como pode ser observado, os extratos subcríticos são ricos em componentes com caráter antioxidante. Pode-se observar que a concentração das antocianinas foi reduzida para cada solvente quando se aumentou a temperatura, indicando que elevadas temperaturas degradam parte das mesmas. Em relação aos demais resultados ( $X_{0}$, FT e AA), o aumento de temperatura leva a melhores rendimentos. Além disso, o tipo de solvente na PLE também afetou a extração de compostos fenólicos e antociânicos. Para FT, AM e AA houve interação significativa $(\mathrm{p}<0,05)$ entre o solvente e a temperatura. Na Tabela 1, uma correlação clara entre o FT e AA pode ser notada. Desta forma, pode-se presumir que resíduos da amora-preta contêm um elevado teor de compostos fenólicos com capacidade antioxidante, e que a AA dos extratos é devida aos fenólicos não antociânicos, já que as antocianinas aparecem em concentrações baixas mesmo em extratos com elevada AA. A partir de uma visão geral dos resultados observados na Tabela 1 a condição de extração PLE considerada a melhor foi aquela em que se utilizou mistura de etanol+água $50 \% \mathrm{v} / \mathrm{v}$ como solvente extrator a temperatura de $100{ }^{\circ} \mathrm{C}$. Nessa condição as variáveis resposta foram as seguintes: $X_{0}=6,33 \%$, FT $=7,36 \mathrm{mg}$ EAG/g RF, AM $=1,02 \mathrm{mg}$ EC3G/g RF, DPPH = 76,03 $\mu \mathrm{mol} \mathrm{ET/g} \mathrm{RF} \mathrm{e} \mathrm{ABTS}=68,28 \mu \mathrm{mol} \mathrm{ET/g} \mathrm{RF}$. Esta condição foi adotada para os experimentos de cinética de PLE.

Tabela 1 - Condições de extração PLE. Resultados para o rendimento global, fenólicos totais, antocianinas monoméricas e atividade antioxidante

\begin{tabular}{ccccccc}
\hline & & & & & \multicolumn{2}{c}{ AA $^{\boldsymbol{d}}$} \\
\cline { 6 - 7 } $\mathbf{T}\left({ }^{\circ} \mathbf{C}\right)$ & Solvente & $\boldsymbol{X}_{\boldsymbol{0}}{ }^{\boldsymbol{}}$ & $\mathbf{F T}^{b}$ & $\mathbf{A M}^{\boldsymbol{c}}$ & $\mathbf{D P P H}$ & ABTS \\
\hline 60 & Água & $3,56 \pm 0.71$ & $2,39 \pm 0.21$ & $0,88 \pm 0.01$ & $14,25 \pm 0.74$ & $21,26 \pm 0.74$ \\
60 & Água pH 2,5 & $12,10 \pm 0.49$ & $1,93 \pm 0.21$ & $0,95 \pm 0.08$ & $12,25 \pm 0.21$ & $31,14 \pm 6.35$ \\
60 & Etanol & $3,23 \pm 0.08$ & $3,18 \pm 0.62$ & $1,25 \pm 0.03$ & $24,33 \pm 4.40$ & $32,04 \pm 6.75$ \\
60 & Etanol (50\%) & $3,85 \pm 0.37$ & $5,23 \pm 0.83$ & $1,40 \pm 0.02$ & $37,04 \pm 2.80$ & $49,24 \pm 3.32$ \\
80 & Água & $4,16 \pm 1.47$ & $3,78 \pm 0.03$ & $0,79 \pm 0.08$ & $33,22 \pm 0.08$ & $36,46 \pm 0.72$ \\
80 & Água pH 2,5 & $14,27 \pm 0.37$ & $4,46 \pm 0.25$ & $0,99 \pm 0.05$ & $36,30 \pm 0.78$ & $43,97 \pm 0.79$ \\
80 & Etanol & $4,23 \pm 0.28$ & $3,72 \pm 0.60$ & $1,39 \pm 0.02$ & $31,40 \pm 4.38$ & $31,48 \pm 5.18$ \\
80 & Etanol (50\%) & $5,19 \pm 0.08$ & $5,51 \pm 0.80$ & $1,08 \pm 0.21$ & $46,38 \pm 1.28$ & $52,10 \pm 3.32$ \\
100 & Água & $6,39 \pm 0.36$ & $4,97 \pm 0.51$ & $0,65 \pm 0.10$ & $42,85 \pm 5.45$ & $42,79 \pm 6.70$ \\
100 & Água pH 2,5 & $14,99 \pm 0.05$ & $5,34 \pm 0.46$ & $0,38 \pm 0.04$ & $40,40 \pm 3.34$ & $51,29 \pm 1.94$ \\
100 & Etanol & $4,46 \pm 0.24$ & $4,12 \pm 0.23$ & $0,93 \pm 0.03$ & $36,61 \pm 0.24$ & $31,07 \pm 4.60$ \\
100 & Etanol (50\%) & $6,33 \pm 0.04$ & $7,36 \pm 0.18$ & $1,02 \pm 0.11$ & $76,03 \pm 1.05$ & $68,28 \pm 2.68$ \\
\hline
\end{tabular}

* Resultados expressos pela média \pm desvio padrão.

${ }^{a}$ Rendimento global de extração expresso em porcentagem $(100 * \mathrm{~g}$ extrato/g RF).

${ }^{\boldsymbol{b}}$ Teor de compostos fenólicos totais expresso em $\mathrm{mg}$ EAG/g RF.

${ }^{c}$ Teor de antocianinas monoméricas expressas em $\mathrm{mg}$ EC3G/g RF.

${ }^{d}$ Atividade antioxidante expressa em $\mu \mathrm{moL} \mathrm{ET/g} \mathrm{RF.}$ 


\subsection{Extração PLE versus Extrações Convencionais}

Os resultados experimentais presentes na Tabela 2 demonstram que o procedimento de PLE é muito mais eficaz na extração de fitoquímicos (maior valor de $X_{0}$ ) e de compostos fenólicos (FT) do resíduo da amora-preta que os realizados por procedimentos convencionais. Além disso, o extrato de PLE apresentou maior atividade antioxidante que a dos outros métodos. Estes resultados podem ser atribuídos à utilização combinada de alta pressão e temperatura durante o processo de extração por PLE. Entretanto, como pode se observar na Tabela 2, o menor teor de antocianinas foi encontrado também na técnica PLE. Esta contradição pode estar relacionada com o procedimento operacional de extração em conjunto com o efeito deletério da temperatura sobre as antocianinas, uma vez que a maceração foi realizada em temperatura ambiente, a extração Soxhlet em temperatura em torno de $40^{\circ} \mathrm{C}$ e a PLE a $100^{\circ} \mathrm{C}$.

Tabela 2 - Resultados obtidos das extrações convencionais e da melhor condição da extração com líquido pressurizado a partir do bagaço da amora-preta

\begin{tabular}{|c|c|c|c|c|c|}
\hline \multirow[b]{2}{*}{ Métodos } & \multirow[b]{2}{*}{$\mathbf{F T}^{a}$} & \multirow[b]{2}{*}{$\mathbf{A M}^{b}$} & \multicolumn{2}{|c|}{ Atividade Antioxidante $^{c}$} & \multirow[b]{2}{*}{$\boldsymbol{X}_{\boldsymbol{0}}^{d}$} \\
\hline & & & DPPH & ABTS & \\
\hline Maceração & $3,66 \pm 0,05^{\mathrm{A}}$ & $1,21 \pm 0,02^{\mathrm{AB}}$ & $29,04 \pm 2,18^{\mathrm{A}}$ & $46,09 \pm 1,15^{\mathrm{A}}$ & $5,02 \pm 0,07^{\mathrm{A}}$ \\
\hline Soxhlet Metanol & $4,64 \pm 0,01^{\mathrm{A}}$ & $1,33 \pm 0,01^{\mathrm{AB}}$ & $39,55 \pm 2,87^{\mathrm{B}}$ & $60,19 \pm 3,30^{\mathrm{B}}$ & $5,25 \pm 0,17^{\mathrm{A}}$ \\
\hline Soxhlet Etanol & $4,10 \pm 0,71^{\mathrm{A}}$ & $1,68 \pm 0,23^{\text {В }}$ & $42,79 \pm 1,19^{\mathrm{B}}$ & $63,10 \pm 4,58^{\text {B }}$ & $5,51 \pm 0,27^{\mathrm{A}}$ \\
\hline PLE & $7,36 \pm 0,09^{\mathrm{B}}$ & $1,02 \pm 0,05^{\mathrm{A}}$ & $76,03 \pm 0,52^{\mathrm{C}}$ & $68,28 \pm 1,34^{\mathrm{B}}$ & $6,23 \pm 0,02^{\mathrm{B}}$ \\
\hline
\end{tabular}

* Resultados expressos pela sua média \pm desvio padrão (DP).

** Letras maiúsculas iguais indicam que não existe diferença entre os métodos de extração, ao nível de $5 \%$ de significância pelo teste Tukey.

${ }^{a}$ Teor de compostos fenólicos totais expresso em mg EAG/g RF.

${ }^{b}$ Teor de antocianinas monoméricas expressas em $\mathrm{mg}$ EC3G/g RF.

${ }^{c}$ Atividade antioxidante expresso em $\mu \mathrm{moL}$ ET/g RF.

${ }^{d}$ Rendimento global de extração expresso em porcentagem $\left(100^{*} \mathrm{~g}\right.$ extrato/g RF).

\subsection{Identificação e Quantificação de Antocianinas por UPLC}

Quatro antocianinas foram simultaneamente e tentativamente identificadas nos extratos do resíduo da amora-preta obtidos por PLE. Foram identificadas como antocianinas majoritárias a cianidina 3-O-glicosídeo (C3G) e a cianidina 3-O-rutinosídeo (C3R) e como minoritárias a cianidina 3-O-malonil-glicosídeo (C3MG) e a cianidina 3-O-dioxalil-glicosídeo (C3DG).

A Tabela 3 mostra a concentração das respectivas antocianinas (C3G, C3R, C3MG e C3DG) presentes em cada condição realizada na PLE. Foi observado que o teor de antocianinas obtidas via UPLC foi inferior quando comparado com os teores obtidos através das análises espectrofotométricas. A hipótese para este comportamento é que a escolha dos padrões avaliados nas análises de UPLC-QToF-MS baseou-se na composição de antocianinas previamente reportadas na amora-preta. Assim, compostos distintos às composições já reportadas, devido a processos de copigmentação com açúcares, ácidos orgânicos ou metais presentes na amostra, não 
podem ser detectados no UPLC devido à falta desses padrões específicos; contudo, é possível que tais compostos estejam sendo detectados colorimetricamente no espectrofotômetro UV-vis.

Tabela 3 - Concentração de antocianinas obtidos nos diferentes ensaios PLE

\begin{tabular}{ccccccc}
\hline $\mathbf{T}\left({ }^{\mathbf{}} \mathbf{C}\right)$ & Solvente & $\mathbf{C 3 G}^{\boldsymbol{a}}$ & $\mathbf{C 3 R}^{b}$ & $\mathbf{C 3 M G}^{\boldsymbol{c}}$ & $\mathbf{C 3 D G}^{\boldsymbol{d}}$ & Total $^{\boldsymbol{e}}$ \\
\hline 60 & Água & $0,42 \pm 0,15$ & $0,05 \pm 0,01$ & $0,01 \pm 0,01$ & $0,02 \pm 0,01$ & $0,50 \pm 0,19$ \\
60 & Água pH 2,5 & $0,76 \pm 0,25$ & $0,07 \pm 0,03$ & $0,02 \pm 0,01$ & $0,03 \pm 0,01$ & $0,88 \pm 0,28$ \\
60 & Etanol & $0,31 \pm 0,08$ & $0,02 \pm 0,01$ & $0,01 \pm 0,01$ & $0,02 \pm 0,01$ & $0,36 \pm 0,09$ \\
60 & Etanol (50\%) & $0,41 \pm 0,02$ & $0,02 \pm 0,01$ & $0,01 \pm 0,01$ & $0,02 \pm 0,01$ & $0,47 \pm 0,03$ \\
80 & Água & $0,49 \pm 0,25$ & $0,05 \pm 0,01$ & $0,02 \pm 0,01$ & $0,02 \pm 0,01$ & $0,57 \pm 0,28$ \\
80 & Água pH 2,5 & $1,05 \pm 0,38$ & $0,09 \pm 0,03$ & $0,02 \pm 0,01$ & $0,03 \pm 0,01$ & $1,21 \pm 0,45$ \\
80 & Etanol & $0,25 \pm 0,01$ & $0,02 \pm 0,01$ & $0,01 \pm 0,01$ & $0,02 \pm 0,01$ & $0,28 \pm 0,01$ \\
80 & Etanol (50\%) & $0,37 \pm 0,03$ & $0,03 \pm 0,01$ & $0,02 \pm 0,01$ & $0,02 \pm 0,01$ & $0,43 \pm 0,04$ \\
100 & Água & $0,34 \pm 0,22$ & $0,04 \pm 0,01$ & $0,01 \pm 0,00$ & $0,01 \pm 0,00$ & $0,41 \pm 0,25$ \\
100 & Água pH 2,5 & $0,27 \pm 0,01$ & $0,04 \pm 0,01$ & $0,01 \pm 0,00$ & $0,01 \pm 0,00$ & $0,32 \pm 0,01$ \\
100 & Etanol & $0,18 \pm 0,01$ & $0,02 \pm 0,01$ & $0,01 \pm 0,00$ & $0,01 \pm 0,0$ & $0,21 \pm 0,01$ \\
100 & Etanol (50\%) & $0,32 \pm 0,04$ & $0.03 \pm 0,00$ & $0,01 \pm 0,00$ & $0,01 \pm 0,00$ & $0,37 \pm 0,04$ \\
\hline
\end{tabular}

*Resultados expressos pela sua média \pm desvio padrão (DP).

${ }^{a}$ Teor de antocianina cianidina 3-O-glicosídeo (C3G) expresso em mg de antocianina (C3G) por grama de RF.

${ }^{b}$ Teor de antocianina cianidina 3-O-rutinosídeo (C3R) expresso em mg de antocianina (C3R) por grama de RF.

${ }^{c}$ Teor de antocianina cianidina 3-O-malonil-glicosídeo (C3MG) expresso em $\mathrm{mg}$ de antocianina (C3MG) por grama de RF.

${ }^{d}$ Teor de antocianina cianidina 3-O-dioxalil-glicosídeo (C3DG) expresso em mg de antocianina (C3DG) por grama de RF.

${ }^{e}$ Teor de antocianinas totais expresso em termos da soma das antocianinas C3G, C3R, C3MG e C3DG por grama de RF.

\subsection{Cinéticas de Extração}

Verifica-se na Figura 1 que, para as três vazões em estudo, até o tempo em torno de 30 minutos há um aumento expressivo do acúmulo de massa, e depois desse tempo o rendimento começa a se estabilizar. Dessa maneira, visualmente é possível afirmar que este é o tempo que caracteriza o período em que a extração dos solutos ocorre predominantemente através da transferência de massa por convecção. Além disso, também é possível verificar que entre o tempo de 30 a 90 minutos há um decréscimo da taxa de transferência de massa, que é resultado da diminuição da área efetiva de contato entre solvente e substrato. Nessa etapa, tanto a transferência de massa por convecção quanto por difusão são importantes na extração. E, aproximadamente a partir de 90 minutos, o rendimento global acumulado (\%) tende a se estabilizar, porque nesse período a retirada de solutos é realizada, predominantemente, pelo mecanismo de difusão. Nesta etapa não há quase nenhum soluto na superfície da amostra. Nota-se ainda que em 120 min de extração o rendimento é maior do que para o tempo adotado de $30 \mathrm{~min}$. Por exemplo, na vazão de $1,67 \mathrm{~mL} / \mathrm{min}$ há um aumento de $6 \%$ para, aproximadamente, $9 \%$. Isso é interessante para o processo, podendo o rendimento ser ainda maior se comparado ás técnicas convencionais, que demandam tempos de extração muito mais elevados. E por fim, pode-se verificar na Figura 1 que 
dentre as vazões trabalhadas a mais interessante seria a de $1,67 \mathrm{~mL} / \mathrm{min}$, já que nesta se gasta uma menor quantidade de solvente no processo de extração e, conjuntamente, é nela que se observa um maior rendimento global em todos os tempos avaliados.

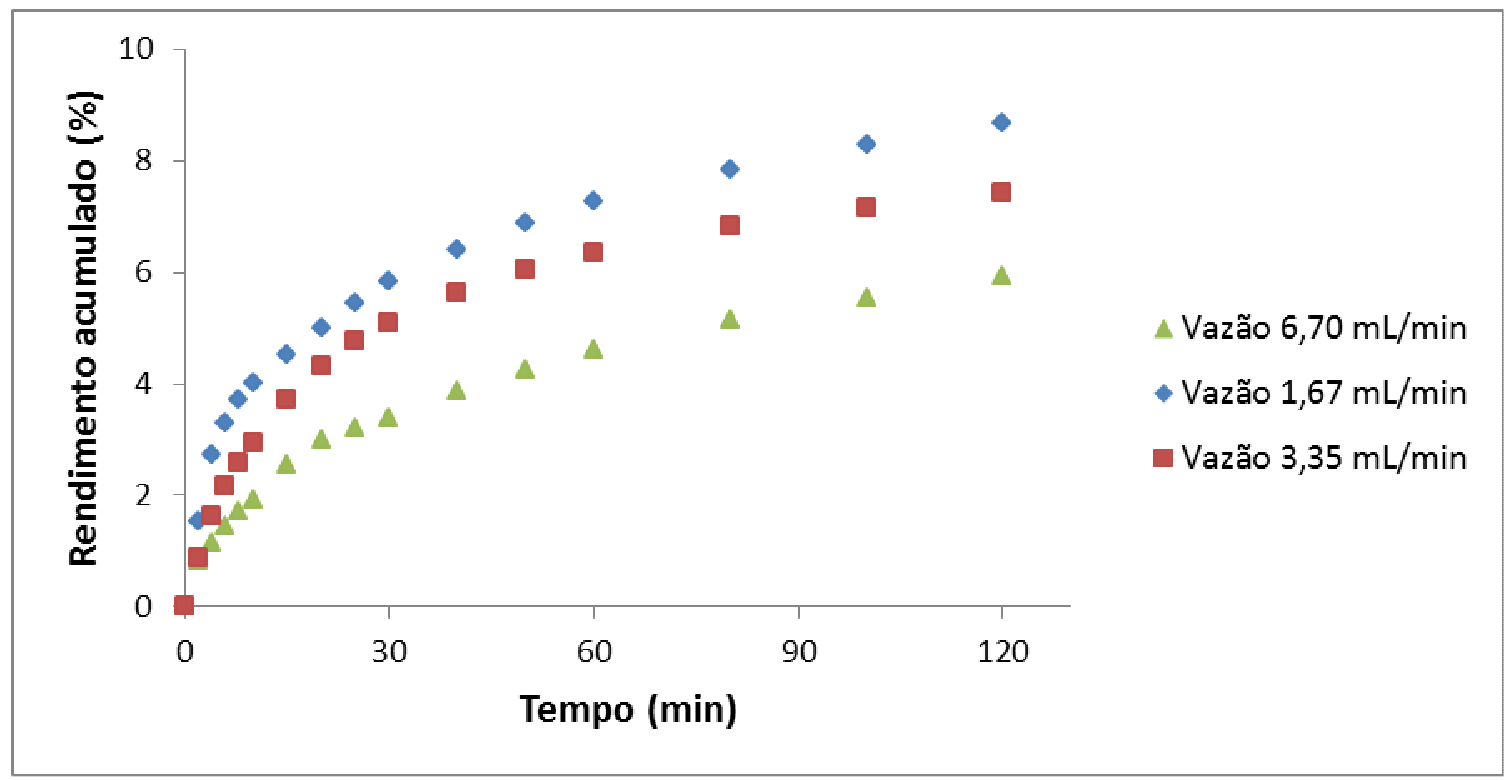

Figura 1 - Curvas globais de PLE de resíduos de amora-preta usando etanol+água (50\% v/v) a $100{ }^{\circ} \mathrm{C}$ em diferentes vazões.

\section{CONCLUSÃO}

Este trabalho mostrou que o bagaço de amora é uma boa fonte de compostos bioativos, como compostos fenólicos e antocianinas, que pode gerar produtos com alta atividade antioxidante a ser aplicados como ingredientes alimentares e cosméticos. Técnicas ambientalmente limpas, como a PLE, são alternativas viáveis frente às extrações convencionais, para a extração de compostos valiosos de bagaço de amora-preta, assim como a partir de resíduos de muitos outros processos alimentares.

\section{REFERENCIAS}

BALASUNDRAM, N.; SUNDRAM, K.; SAMMAN, S. Phenolic compounds in plants and agro industrial by-products: Antioxidant activity, occurrence, and potential uses. Food Chem., v. 99, p. 191-203, 2006.

BRAND-WILLIAMS, W.; CUVELIER, M. E.; BERSET, C. Use of a free radical method to evaluate antioxidant activity. LWT - Food Sci. Technol., v. 28, p. 25-30, 1995.

GIUSTI, M. M.; WROLSTAD, R. E. Characterization and measurement of anthocyanins by uv- 
visible spectroscopy. In: (Ed.). Current Protocols in Food Analytical Chemistry: John Wiley \& Sons, Inc., 2001.

KAUME, L.; HOWARD, L. R.; DEVAREDDY, L. The blackberry fruit: a review on its composition and chemistry, metabolism and bioavailability, and health benefits. J. Agric. Food Chem., v. 60, p. 5716-5727, 2011.

MUSTAFA, A.; TURNER, C. Pressurized liquid extraction as a green approach in food and herbal plants extraction: a review. Anal. Chim. Acta, v. 703, p. 8-18, 2011.

RE, R.; PELlEGRINI, N.; PROTEGGENTE, A.; PANNALA, A.; YANG, M.; RICE-EVANS, C. Antioxidant activity applying an improved ABTS radical cation decolorization assay. Free Radical Biol. Med., v. 26, p. 1231-1237, 1999.

SINGLETON, V. L.; ORTHOFER, R.; LAMUELA-RAVENTÓS, R. M. Analysis of total phenols and other oxidation substrates and antioxidants by means of folin-ciocalteu reagent. In: LESTER, P. (Ed.). Methods in Enzymology: Academic Press, v. 299, p.152-178, 1999.

SINGLETON, V. L.; ROSSI, J. A. Colorimetry of total phenolics with phosphomolybdicphosphotungstic acid reagents. Am. J. Enol. Vitic., v. 16, p. 144-158, 1965.

WIJNGAARD, H; HOSSAIN, M. B.; RAI, D. K.; BRUNTON, N. Techniques to extract bioactive compounds from food by-products of plant origin. Food Res. Int., v. 46, p. 505-513, 2012. 